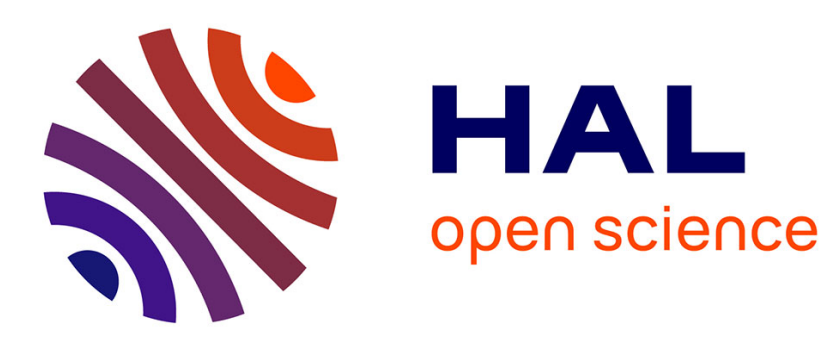

\title{
Améliorer l'auto-efficacité collective des équipes de cadres formateurs en IFSI par la didactique professionnelle
}

\author{
Marc Nagels
}

\section{- To cite this version:}

Marc Nagels. Améliorer l'auto-efficacité collective des équipes de cadres formateurs en IFSI par la didactique professionnelle. Recherche en soins infirmiers, 2011, 104, pp.0. hal-00565412

\section{HAL Id: hal-00565412 \\ https://hal.science/hal-00565412}

Submitted on 12 Feb 2011

HAL is a multi-disciplinary open access archive for the deposit and dissemination of scientific research documents, whether they are published or not. The documents may come from teaching and research institutions in France or abroad, or from public or private research centers.
L'archive ouverte pluridisciplinaire HAL, est destinée au dépôt et à la diffusion de documents scientifiques de niveau recherche, publiés ou non, émanant des établissements d'enseignement et de recherche français ou étrangers, des laboratoires publics ou privés. 


\title{
Améliorer l'auto-efficacité collective des équipes de cadres formateurs en IFSI par la didactique professionnelle
}

\author{
Marc NAGELS \\ 17 mars Conseil \\ CREAD Université Rennes 2
}

\section{Résumé}

Le but de l'étude est de montrer que l'analyse de l'activité est un facteur de construction de l'auto-efficacité collective des formateurs de cinq instituts de formation en soins infirmiers (IFSI).

En tant que système de croyances collectif sur la capacité du groupe à atteindre ses objectifs, l'auto-efficacité trouve ses fondements dans la théorie sociocognitive qui articule, au sein d'une causalité triadique réciproque, les facteurs personnels, comportementaux et environnementaux de l'agentivité humaine. Les déterminants personnels (auto-efficacité, organisation cognitive de l'activité) sont mis en relation avec les facteurs comportementaux (production de la performance) et environnementaux (attribution de compétence, tâches et normes professionnelles).

L'intervention a consisté à initier cent neuf cadres formateurs à l'analyse du travail dans un contexte de réformes hospitalières. L'auto-efficacité collective des formateurs apparait comme une variable clé du succès de ces réformes. L'auto-efficacité collective a fait l'objet d'un pré test et d'un post test qualitatif sur la base d'indicateurs motivationnels, de l'effectivité des réalisations pédagogiques à destination des étudiants et de l'estimation de la performance du groupe à conduire la réforme.

Le niveau d'auto-efficacité collective augmente et les premières réalisations entretiennent la croyance des formateurs dans leur capacité à réussir collectivement la mise en œuvre du plan de formation.

Il s'agit ici de la première recherche en France qui montre que l'analyse de l'activité s'accompagne d'une augmentation de l'auto-efficacité collective au travail. Ces résultats interrogent les pratiques des formateurs au-delà du seul secteur paramédical.

Mots clés: Analyse de l'activité, didactique professionnelle, auto-efficacité collective, évaluation des compétences, formation en soins infirmiers, compétence. 
La notion de compétence professionnelle est située au cœur de la réforme de la formation en soins infirmiers

Former les compétences ne s'improvise pas tout à fait, c'est l'objet même de la didactique professionnelle, mais les cadres formateurs et les soignants qui ont été formés aux méthodes de la didactique professionnelle sont peu nombreux aujourd'hui. Le projet de la didactique professionnelle est celui de l'analyse du travail pour la formation ${ }^{(1)}$. Elle a pour objet l'analyse de l'activité pour repérer les besoins préalablement à la conception d'une action de formation. L'analyse de l'activité est également une ressource privilégiée pour concevoir et organiser des séquences d'apprentissage. A ce titre, la diffusion de méthodes de type didactique professionnelle représente bien une condition de réussite de la réforme, une condition centrale, au cœur même de ses finalités.

Il est une autre condition de réussite : celle de l'engagement des individus et des équipes dans la réforme. De quelles ressources professionnelles, mais aussi personnelles, disposent-ils pour s'adapter? Les formateurs disposent-ils d'une auto-efficacité collective suffisante pour se mobiliser, concevoir les changements de pratiques pédagogiques à venir et réorienter explicitement leur action vers la formation des compétences ?

Ainsi, en période de réforme, l'analyse de l'auto-efficacité individuelle et de l'auto-efficacité collective aide à mieux comprendre les obstacles aux changements. Cela nous conduit à proposer les résultats d'une recherche-action qui met en relation l'auto-efficacité collective et l'analyse de l'activité dans le cadre de la didactique professionnelle en soins infirmiers. L'hypothèse est que l'auto-efficacité individuelle et collective des cadres formateurs augmenterait à la suite d'une formation - sensibilisation à la didactique professionnelle.

Notre problématique vise à articuler deux ensembles conceptuels : premièrement, celui de compétence, vu sous l'angle de l'organisation cognitive de l'activité, deuxièmement, l'autoefficacité collective, variable déterminant les comportements d'engagement des formateurs dans la réforme.

Nous décrirons successivement ces deux ensembles avant de poser par hypothèse que l'acquisition d'une méthode d'analyse de l'activité développerait l'auto-efficacité collective des équipes en IFSI.

\section{Un modèle élargi de la compétence}

Le concept de compétence articule deux dimensions, interne et externe à l'individu. La première s'intéresse à l'organisation cognitive de l'activité. Cette organisation reste largement implicite pour l'individu mais elle devient accessible par un effort d'explicitation à l'aide de méthodes d'analyse de l'activité. Nous pouvons la comprendre comme une mise en réseau d'éléments épars, de ressources cognitives diversifiées qui ne sont pas toutes des savoirs, au sens académique du terme. La deuxième dimension est de nature psychosociale, la compétence est un jugement, une évaluation de la performance, mais c'est la fabrication sociale du jugement qui nous intéresse ici sans examiner vraiment la pertinence de l'organisation cognitive de l'activité qui conduit à la performance évaluée.

Dans une certaine mesure, la performance et la compétence sont disjointes mais il arrive qu'en matière d'évaluation de la compétence, des professionnels confondent souvent l'évaluation de la performance, ce que l'individu fait et comment il le fait, et l'évaluation de la compétence, c'est-à-dire le jugement social porté sur la performance ${ }^{(2)}$. L'une signe l'efficacité de l'individu à travers ses actions, l'autre l'appréciation sociale de l'efficacité. Or, 
ces deux évaluations ne renvoient pas au même ordre de faits. D'ailleurs, les cadres formateurs recherchent explicitement la genèse cognitive des étudiants à travers le développement de leurs schèmes et de leurs modèles opératifs ${ }^{(3)}$ alors, qu'en entreprise, les individus compétents disposent, certes, de schèmes pour réguler efficacement leur activité mais ils font aussi preuve de clairvoyance normative (la connaissance du système de normes et de contre normes) pour identifier les habitudes professionnelles et se faire reconnaitre comme compétent, sur la base de leurs performances. La clairvoyance est une compétence qui permet le repérage de la valorisation des explications internes et de la dévalorisation des explications externes ${ }^{(4)}$.

L'individu est efficace lorsqu'il dispose, au plan cognitif, d'une organisation de son activité qui lui permet de produire la performance visée, mais cette performance sera-t-elle appréciée positivement par son environnement professionnel? Sera-t-il «compétent», i.e. sa performance sera-t-elle évaluée et jugée conforme aux attentes de son environnement social ? La dimension externe de la compétence rend compte de l'interaction entre l'individu et son environnement dans le cadre des évaluations de compétence. La compétence devient alors une signification partagée, support aux jugements sociaux et aux attributions d'efficacité.

Ce modèle trouve ses fondements dans la théorie sociocognitive qui articule, au sein d'une causalité triadique réciproque, les facteurs personnels, comportementaux et environnementaux de l'agentivité humaine ${ }^{(5)}$. Ce modèle général de la compétence met en relation les déterminants personnels (auto-efficacité, organisation cognitive de l'activité) avec les facteurs comportementaux (production de la performance) et environnementaux (attribution de compétence, tâches et normes professionnelles). L'inspiration de ce modèle général tire sa force des convergences de la théorie sociocognitive avec l'analyse de l'activité du point de vue de la didactique professionnelle ${ }^{(1)}$. La figure 1 indique l'articulation des concepts qui fondent ce modèle.

Fig. 1 Un modèle général de la compétence

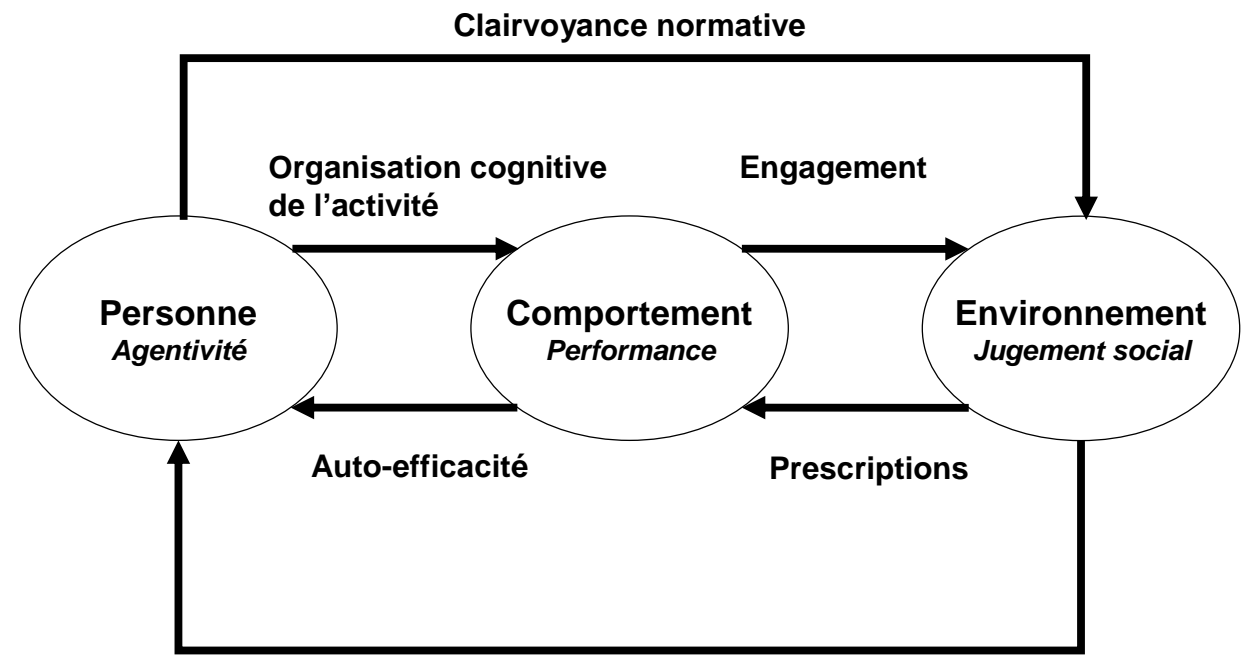

Evaluation

Dans le cadre de cet article, nous ne retiendrons que la première dimension cognitive, considérant que l'ingénierie pédagogique des formateurs en IFSI vise d'abord l'augmentation de l'efficacité soignante des étudiants. En effet, les formateurs sont très sensibles à cette dimension cognitive sur laquelle ils peuvent agir directement.

L'activité est organisée et sous-tendue par un schème, c'est-à-dire « une organisation invariante de la conduite pour une classe de situations ${ }^{(6)}$. 
La compétence, comme le propose la figure 2, schéma destiné aux cadres formateurs pendant l'intervention, ne consiste bien évidemment pas à reproduire indéfiniment les mêmes modes opératoires. Au contraire, l'individu, par et dans l'activité, s'adapte à des situations toujours singulières. Ce sont ces ressources de conceptualisation et d'adaptation qu'il convient de repérer pour décrire la compétence.

Fig. 2 La compétence du point de vue cognitif : invariance et adaptation de l'activité

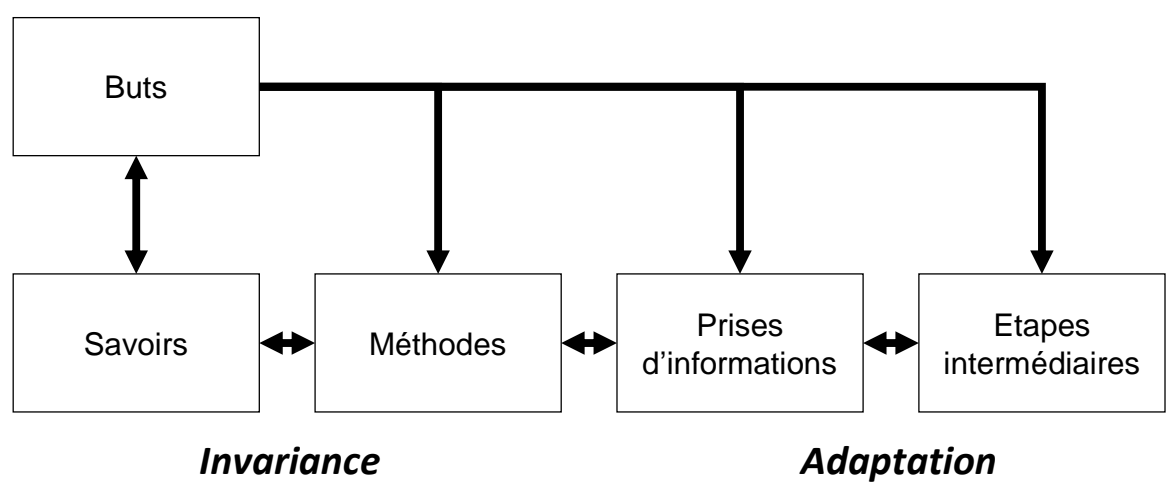

Á l'intérieur d'une classe de situations données, l'invariance se manifeste et fait l'objet d'un repérage par l'individu, ce qui lui permet d'agir aussi avec constance et régularité. Le sujet évalue les variables situationnelles, estime ses capacités et la valeur accordée aux performances obtenues ${ }^{(7)}$.

La construction d'une compétence suppose que l'individu ait connu des expériences professionnelles variées et nombreuses et qu'il les ait analysées. Ses pratiques professionnelles en ont été modifiées et il a gagné en efficacité. Qu'a-t-il appris au fil de ses expériences successives ? Essentiellement à mieux réguler son action, à l'organiser de manière plus performante. Tous les registres de l'activité se développent : « les gestes, les activités intellectuelles et techniques, l'énonciation et le langage, l'interaction sociale et l'affectivité $\gg{ }^{(8)}$.

\section{L'auto-efficacité individuelle et collective, variable-clé de l'agentivité}

La maîtrise d'une compétence renforce le sentiment de l'individu qu'il peut agir efficacement dans des conditions difficiles, ce qui signifie que la compétence contribue à la construction et au développement de l'agentivité humaine. L'agentivité se définit comme la capacité d'intervention sur les autres et le monde où les individus et les groupes sont tout à la fois les producteurs et les produits des systèmes sociaux. En fait, «l'agentivité humaine se caractérise par plusieurs aspects majeurs qui opèrent consciemment de manière fonctionnelle et phénoménale. Il s'agit de l'intentionnalité et de l'anticipation, qui permettent une extension temporelle de l'agentivité, de l'autorégulation par l'influence réactive sur soi-même, et de l'autoréflexion sur ses capacités personnelles, la qualité de son propre fonctionnement et le sens et la finalité des entreprises de son existence ${ }^{(9)}$.

L'agentivité se manifeste de trois manières différentes : il s'agit tout d'abord de l'effet direct de l'intervention de la personne mais elle peut opérer aussi par le biais d'une "procuration » 
par laquelle le sujet escompte l'action d'autrui pour atteindre les buts souhaités. Enfin, l'agentivité peut être collective, par l'intermédiaire d'une coordination des efforts d'un groupe. L'agentivité se comprend à l'intérieur d'un modèle du sujet qui négocie systématiquement ses comportements, ses motivations et ses affects avec son environnement physique ou social $^{(10)}$.

La théorie sociale cognitive décrit l'auto-efficacité comme la "variable clé » de l'agentivité humaine ${ }^{(5)}$. Elle est définie ainsi : « la croyance de l'individu en sa capacité d'organiser et d'exécuter la ligne de conduite requise pour produire des résultats souhaités ». L'autoefficacité est un facteur majeur de l'apprentissage en favorisant le pilotage métacognitif des apprentissages. Les apprenants qui obtiennent les meilleurs résultats sont ceux qui se fixent plus d'objectifs, ou des objectifs plus ambitieux, utilisent plus de stratégies pour étudier, pilotent attentivement la progression de leurs apprentissages et optimisent leurs efforts en vue d'atteindre les résultats souhaités ${ }^{(11)}$.

Il est à noter que l'auto-efficacité individuelle «ne mesure pas les aptitudes mais exprime ce que l'individu croit pouvoir faire dans des situations variées ${ }^{(12)}$. Comme le définit Albert BANDURA : "L'efficacité personnelle perçue concerne la croyance de l'individu en sa capacité d'organiser et d'exécuter la ligne de conduite requise pour produire des résultats souhaités ${ }^{(5)}$. Il se trouve que les personnes agissent quand elles pensent que leurs actions vont produire les performances désirées, sans toutefois les confondre avec les conséquences produites par les performances. Sinon, elles évitent les tâches difficiles, réduisent leur persévérance ou renoncent plus facilement.

Dans le cas qui nous occupe ici, celui de la mise en œuvre de la réforme de la formation en soins infirmiers, l'auto-efficacité individuelle se combine avec l'auto-efficacité collective. Le sentiment d'efficacité collective est « une croyance partagée par un groupe en ses capacités conjointes d'organiser et d'exécuter les actions nécessaires pour produire un niveau donné de réalisations (...) L'efficacité collective n'est pas simplement la somme des croyances des individus en leur efficacité, mais il s'agit plutôt d'une caractéristique émergente du groupe, produite par des dynamiques coordonnées et interactives $\gg{ }^{(5)}$.

L'auto-efficacité collective est définie comme «la croyance collective du groupe qu'il peut exécuter une tâche spécifique avec succès $»{ }^{(13)}$. Les capacités opérationnelles du groupe sont au cœur de l'auto-efficacité collective qui représente un système de croyances partagée «par un groupe sur ses capacités conjointes d'organiser et d'exécuter les actions nécessaires pour produire un niveau donné de réalisations » ${ }^{(5)}$.

Les facteurs de construction de l'auto-efficacité collective sont les mêmes que ceux de l'autoefficacité individuelle, notamment la maîtrise de situations de réussite, l'observation vicariante et la persuasion sociale. Néanmoins, les particularités liées à la situation collective engagent à ajouter d'autres variables : la taille du groupe, le leadership et la cohésion du groupe $^{(14)}$.

Le niveau de croyances d'efficacité collective est par ailleurs un bon prédicteur de la performance d'un groupe, tout en considérant que cette variable du comportement humain ne représente effectivement que «la perception de la probabilité que l'effort commun aboutira à la réussite collective » ${ }^{(15)}$. Plusieurs effets sont associés à un niveau élevé d'auto-efficacité collective. En premier lieu, l'efficacité d'un groupe est lié à la croyance collective que le groupe est et sera productif ${ }^{(16)}$; la désirabilité du groupe et sa cohésion augmentent en conséquence $^{(17)}$. En fait, le niveau d'auto-efficacité collective influence les réalisations du groupe à travers les stratégies qu'il mettra en œuvre, sa persévérance face aux difficultés et la quantité d'énergie qu'il pourra mobiliser.

D'autre part, des études ont montré l'influence positive de l'efficacité collective des enseignants sur leur capacité à concevoir des dispositifs d'autoformation éducative ainsi que sur la performance scolaire des élèves ${ }^{(18)}$. L'auto-efficacité collective est dépendante des 
perceptions que les enseignants, les parents et les membres du personnel entretiennent sur eux-mêmes mais elle influe également sur les perceptions d'auto-efficacité individuelle, sur l'engagement affectif et la satisfaction au travail ${ }^{(19)}$.

De plus, il semble que le stress professionnel, lié à un contexte d'incertitude puisse être prévenu par un fort sentiment d'efficacité individuelle et collective ${ }^{(20)}$. Le stress apparaît comme une problématique préoccupante à laquelle les organisations doivent faire face. Cinq grandes catégories de facteurs organisationnels et psychosociaux sont susceptibles de générer du stress : la situation macro-économique avec ses exigences de productivité, l'environnement matériel, les évolutions sociologiques de la relation à la clientèle ou l'utilisation des techniques de l'information et de la communication, l'organisation du travail et la qualité des relations de travail ${ }^{(21)}$. L'auto-efficacité peut jouer un rôle de contrôle déterminant sur les trois derniers facteurs, au moins. Nous noterons que, peu ou prou, la réforme de la formation en soins infirmiers peut s'inscrire dans chaque catégorie de facteurs, le stress ressenti et exprimé par un certain nombre d'équipes de cadres formateurs devient compréhensible.

Le secteur sanitaire est particulièrement touché par le stress au travail ainsi que le souligne une étude menée en 2005 par la fondation de Dublin dans l'union européenne. Plus particulièrement encore, les professions soignantes sont touchées par le stress, ce qui conduit à des abandons prématurés du métier. L'épuisement émotionnel est particulièrement désigné comme une cause probable ${ }^{(22)}$, ce qui met l'accent sur l'importance d'un collectif de travail pluridisciplinaire soudé ${ }^{(23)}$.

Les soignants disent subir des organisations du travail perçues comme toujours plus efficientes mais qui laissent moins de place à la relation au patient ou aux collègues. Ils déplorent le peu de contrôle qu'ils peuvent exercer sur leurs situations de travail («l'institution décide de tout») et ils estiment que dans une situation donnée, la probabilité d'apparition d'un événement est la même quelle que soit l'action qu'ils peuvent entreprendre. Cette absence de contrôle renvoie au sentiment de l'individu que ses actions ne pourront modifier le cours des événements et donc à une faible auto-efficacité individuelle ou collective. Il est donc possible d'interpréter ces abandons prématurés sous l'angle de la résignation acquise ou de l'impuissance apprise ${ }^{(24)}$. La résignation apprise a pour effet de dégrader les performances futures des individus, de faire obstacle au développement professionnel et de modifier l'humeur du sujet.

La variable de l'auto-efficacité collective est une variable composite qui permet de prédire un certain nombre de comportements collectifs d'engagement dans l'action, de réduction de la flânerie sociale, d'une éventuelle résignation apprise et du stress au travail. Elle nous intéresse particulièrement ici quant à la capacité des équipes de cadres formateurs en IFSI à produire avant la fin 2009 un nouveau projet de formation à la compétence pour les trois années de formation infirmière. Dans un contexte de réformes hospitalières incessantes et d'une réingénierie des formations paramédicales, l'auto-efficacité collective appliquée à la formation des compétences apparait comme une variable clé du succès de ces réformes.

Les études dans les milieux soignants qui éclairent les compétences infirmières sont peu nombreuses. Certaines, pourtant, abordent conjointement ou séparément les questions d'analyse de l'activité et d'auto-efficacité ${ }^{(25)}$. Nous nous inscrirons dans cette série d'études mais nous noterons qu'à la différence de l'auto-efficacité individuelle, l'auto-efficacité collective et sa relation avec l'analyse de l'activité ont été peu explorées jusqu'à présent.

Nous sommes ici devant une vaste problématique, celle de l'apprentissage des adultes, apprentissage des formateurs, apprentissage des étudiants, laquelle a souvent été pensée, dans la formation en soins infirmiers, sous l'angle du rapport au savoir, savoir à transmettre, savoir à acquérir : «Comment les individus arrivent-ils à donner du sens à ce qu'ils apprennent ? Comment peut-on, en tant qu'enseignant ou formateur, aider les apprenants à donner du sens 
au savoir enseigné ? Quels sont les facteurs qui conditionnent une «transmission de connaissances » réussie ? ${ }^{(26)}$.

Vaste problématique qui articule développement des compétences des formateurs et développement des compétences des étudiants, ce qui dépasse le strict rapport au savoir. Certes, apprendre suppose de «donner du sens », mais, dans le cas de la construction des compétences, que s'agit-il d'apprendre ? Acquérir une compétence suppose de comprendre tout à la fois une situation et les moyens d'agir efficacement sur la situation, c'est-à-dire de partager une signification avec autrui. La compréhension suppose l'interprétation de la réalité «selon des critères communs. A partir des ses expériences antérieures qui orientent la perception, on doit arriver à distinguer des éléments explicites d'une situation et à en inférer ceux qui sont implicites. Il est également important de percevoir la façon dont ces éléments sont reliés entre eux ${ }^{(26)}$. Les cadres théoriques décrits précédemment, ceux de l'analyse de l'activité et de l'agentivité, permettent de relier l'expérience, la motivation, le savoir-faire et l'action collective. C'est pourquoi, le but de cette recherche-action est de déterminer les facteurs spécifiques à la construction de l'auto-efficacité collective dans ce contexte particulier. Nous posons par hypothèse qu'un travail en équipe d'apprentissage des principaux concepts et méthodes de la didactique professionnelle représente une source de construction de l'auto-efficacité collective appliquée à la formation professionnelle dans une approche par compétences.

\section{Le dispositif empirique}

Notre hypothèse suppose une relation entre la formation à l'analyse du travail et l'augmentation de l'auto-efficacité collective des cadres formateurs pour mettre en application la réforme.

Le dispositif empirique a été mené dans les conditions «naturelles » d'une recherche-action en IFSI et non en laboratoire, ce qui aurait permis de contrôler plus rigoureusement les variables.

Nous décrirons de quelle manière nous avons mesuré l'auto-efficacité collective et comment s'est déployée la formation à l'analyse du travail dans une perspective de didactique professionnelle.

\section{Présentation de la population}

La recherche action a été menée en 2008 et 2009 au sein de cinq IFSI de plusieurs régions de France métropolitaine. Certains IFSI, ou d'autres interventions sur le même thème à l'occasion de journées professionnelles, n'ont pas été pris en compte: le nombre de formateurs ou de directeurs d'IFSI présents était d'environ 300 mais aucune mesure de l'autoefficacité collective ne pouvait être réalisée.

Au total, la population retenue est de cent neuf formateurs, comme le détaille le tableau 1. Les formateurs ont été initiés à l'analyse du travail et à la didactique professionnelle par des interventions de type formation continue en intra-établissement. La durée de l'intervention était variable, étalée de quelques semaines ou de quelques mois, sur des journées ou des demijournées. En tout état de cause, l'intervention n'a jamais excédé huit journées complètes.

Tableau 1 Présentation de la population 


\begin{tabular}{|c|c|c|}
\hline IFSI & Nombre de cadres formateurs & Nombre d'interventions \\
\hline A & 15 & 9 \\
\hline B & 20 & 8 \\
\hline C & 30 & 5 \\
\hline D & 20 & 8 \\
\hline E & 24 & 4 \\
\hline
\end{tabular}

Le tableau 2 indique que la population concernée par la recherche-action ne diffère pas sensiblement d'autres populations de cadres formateurs en IFSI. Ces données sont extraites de deux études non publiées, conduites en 2008 par des directions régionales de l'action sanitaire et sociale. Les régions 1 et 2 rassemblent un grand nombre de cadres formateurs.

Tableau 2 Comparaison de la population avec des formateurs issus d'autres régions

\begin{tabular}{|c|c|c|c|c|c|c|c|}
\hline & $\begin{array}{l}\text { Nombre de } \\
\text { formateurs } \\
\text { permanents }\end{array}$ & $\begin{array}{c}\text { Moyenne } \\
\text { d'âge }\end{array}$ & Dipl & $\begin{array}{l}\text { adre de } \\
\text { é }\end{array}$ & $\begin{array}{r}\text { Diplôm } \\
\text { le pl } \\
\mathbf{f c} \\
\text { uni }\end{array}$ & $\begin{array}{l}\text { versitaire } \\
\text { evé ( } \mathrm{si} \\
\text { ion } \\
\text { aire) }\end{array}$ & $\begin{array}{l}\text { Formateurs } \\
\text { sans diplôme } \\
\text { universitaire }\end{array}$ \\
\hline \multirow{6}{*}{$\begin{array}{l}\text { Population de } \\
\text { la recherche- } \\
\text { action }\end{array}$} & \multirow[t]{6}{*}{109} & \multirow[t]{6}{*}{47 ans } & \multirow[t]{3}{*}{ Oui } & \multirow[t]{3}{*}{$91 \%$} & DU & $19,74 \%$ & \multirow[t]{6}{*}{$30,28 \%$} \\
\hline & & & & & DEUG & $2,63 \%$ & \\
\hline & & & & & Licence & $44,74 \%$ & \\
\hline & & & \multirow[t]{3}{*}{ Non } & \multirow[t]{3}{*}{$9 \%$} & Master 1 & $18,42 \%$ & \\
\hline & & & & & Master 2 & $14,47 \%$ & \\
\hline & & & & & Doctorat & $0,00 \%$ & \\
\hline \multirow[t]{6}{*}{ Région 1} & \multirow[t]{6}{*}{330} & \multirow[t]{6}{*}{49 ans } & \multirow[t]{3}{*}{ Oui } & \multirow[t]{3}{*}{$92 \%$} & DU & $30,15 \%$ & \multirow[t]{6}{*}{$57,11 \%$} \\
\hline & & & & & $\overline{\text { DEUG }}$ & $1,76 \%$ & \\
\hline & & & & & Licence & $34,92 \%$ & \\
\hline & & & \multirow[t]{3}{*}{ Non } & \multirow[t]{3}{*}{$8 \%$} & Master 1 & $24,12 \%$ & \\
\hline & & & & & Master 2 & $7,79 \%$ & \\
\hline & & & & & Doctorat & $1,26 \%$ & \\
\hline \multirow[t]{6}{*}{ Région 2} & \multirow[t]{6}{*}{926} & \multirow[t]{6}{*}{48 ans } & \multirow[t]{3}{*}{ Oui } & \multirow[t]{3}{*}{$87 \%$} & DU & $10,08 \%$ & \multirow[t]{6}{*}{$24,85 \%$} \\
\hline & & & & & DEUG & $4,84 \%$ & \\
\hline & & & & & Licence & $60,89 \%$ & \\
\hline & & & \multirow[t]{3}{*}{ Non } & \multirow[t]{3}{*}{$13 \%$} & Master 1 & $10,48 \%$ & \\
\hline & & & & & Master 2 & $13,31 \%$ & \\
\hline & & & & & Doctorat & $0,40 \%$ & \\
\hline
\end{tabular}

La moyenne d'âge de la population de la recherche-action est proche de celle des autres régions, de même que la proportion de cadres de santé diplômés. Les non diplômés sont essentiellement des infirmiers faisant fonction de cadre, ils interviennent dans la formation des aides-soignants.

Même si la recherche-action avait comme objectif d'anticiper sur la réforme de la formation en soins infirmiers, des formateurs d'aides-soignants y participaient pour deux raisons principales : premièrement, la formation des aides-soignants a fait l'objet récemment d'une réingénierie pour promouvoir l'approche par compétences, les formateurs des aides-soignants disposaient d'une expérience à ce sujet et se sont révélés disponibles pour la communiquer ; deuxièmement, la volonté des directeurs des IFSI-IFAS était de former ensemble la totalité des équipes.

Le niveau de formation universitaire parmi la population considérée est comparable à celui des populations prises en référence. 


\section{Mesure et appréciation de l'auto-efficacité collective au travail}

Il existe deux manières de mesurer l'auto-efficacité collective. La première consiste à additionner les évaluations d'efficacité au travail de chaque membre du groupe ; la deuxième additionne les évaluations d'efficacité que font les membres des capacités de leur groupe pris comme un tout ${ }^{(5)}$. Les deux types de mesure sont également valables puisque l'auto-efficacité individuelle et l'auto-efficacité collective sont relativement interdépendantes. Quand un individu évalue son auto-efficacité personnelle, en situation collective du moins, il prend en compte les processus collectifs qui facilitent ou font obstacle à sa propre performance.

\section{L'échelle d'auto-efficacité au travail}

L'échelle que nous avons reprise pour les besoins de cette recherche mesure l'auto-efficacité collective au travail. Elle s'intéresse surtout au niveau de l'auto-efficacité en référence à des obstacles possibles, mais aussi à la force de l'auto-efficacité face à des événements inattendus et difficiles à régler et à la généralisation de l'auto-efficacité à l'intérieur d'une classe d'activités liées à la formation en soins infirmiers.

Cette échelle a d'abord été conçue et utilisée dans le cadre de la recherche «Optimiser les apprentissages professionnels informels » conduite en association avec le Centre de Recherche Éducation et Formation de l'Université Paris-Ouest Nanterre La Défense et Interface Recherche ${ }^{(27)}$. Une thèse récente s'était intéressée à l'auto-efficacité collective de soignantes ${ }^{(28)}$, la même échelle avait été adaptée pour mesurer l'auto-efficacité collective, en transformant le «je » en «nous ». En ce qui nous concerne, nous avons simplement veillé à ce que les énoncés de l'échelle renvoient effectivement à une pratique de formateurs en IFSI et non à une pratique soignante dans un service de soins.

L'échelle d'auto-efficacité est une échelle de type Likert. Elle comprend dix énoncés de forme déclarative orientés positivement, se référant à des comportements spécifiques en milieu professionnel. Ils sont suivis de réponses indiquant plusieurs niveaux d'accord possibles : «tout à fait d'accord », «plutôt d'accord », «pas vraiment d'accord », «pas du tout d'accord ». Une seule réponse est possible par énoncé et il n'y a pas de point milieu.

Nous avons décidé d'additionner les scores individuels de chaque membre des équipes pour ensuite établir le taux d'efficacité collective de l'équipe sur la même échelle de valeurs, à savoir de 10 points, auto-efficacité minimale, à 40 points, auto-efficacité maximale. L'autoefficacité au travail a fait l'objet d'une mesure avant et après l'intervention.

\section{L'appréciation qualitative de l'auto-efficacité collective}

L'auto-efficacité collective a également fait l'objet d'un prétest et d'un posttest qualitatif Nous nous sommes intéressés au niveau d'auto-efficacité collective en référence aux situations de formation proposant une acquisition de compétences : cours magistraux, études de cas, laboratoire de simulation, reprise d'alternance, etc. Dans ces situations, les différents formateurs apportent des ressources, se concertent et interagissent. Plusieurs paramètres sont enregistrés, il s'agit d'indices qualitatifs recueillis dans les discours : le partage des croyances, la coordination du groupe, les ressources collectives ${ }^{(14)}$. L'auto-efficacité collective a été évaluée à l'aide d'indicateurs motivationnels tels que l'engagement, les construits motivationnels, les facteurs individuels et situationnels ${ }^{(29)}$, de l'effectivité des réalisations 
pédagogiques à destination des étudiants et de l'estimation de la performance du groupe à conduire la réforme.

\section{Une formation à la didactique professionnelle}

Les mesures et l'appréciation de l'auto-efficacité collective encadraient une intervention destinée à sensibiliser les formateurs à la didactique professionnelle et leur faire acquérir les ressources nécessaires au changement de pratiques pédagogiques.

Même si l'objet de la didactique professionnelle est particulièrement clair : l'analyse de l'activité pour former les compétences, pourquoi choisir d'initier les formateurs à la didactique professionnelle plutôt qu'à l'ergonomie ? Une formation à l'ergonomie qui s'intéresse aussi depuis longtemps aux compétences, n'aurait-elles pas permis d'atteindre les objectifs? Il se trouve que l'ergonomie est l'une des trois influences majeures de la didactique professionnelle, à côté d'une approche cognitive de l'activité et de la didactique des disciplines. La didactique professionnelle s'est constituée de manière originale en intégrant ces trois influences et en s'orientant explicitement vers une démarche opérationnelle de formation professionnelle des adultes. De son côté, l'ergonomie, examine également les «interactions sur lesquelles il serait possible d'agir pour modifier les comportements » ${ }^{(30)}$ mais, en secteur sanitaire, l'ergonomie peut étudier préférentiellement le temps passé au lit du patient et mettre en évidence des contraintes à gérer, contraintes physiques, cognitives et émotionnelles. Son projet n'est pas spécifiquement de former les compétences des soignants mais plutôt d'agir sur les conditions de travail. Par exemple, l'ergonomie en étudiant le syndrome de surmenage professionnel a pu montré remarquablement «l'isolement du personnel et le manque de renforcement et de gratification des soignants par la hiérarchie » (31)

Former à la didactique professionnelle est plus efficace au vu des objectifs annoncés ici parce que l'intervention vise le renforcement pédagogique dans le cadre de la réforme et poursuit la finalité explicite du développement des compétences des formateurs. La conséquence attendue est la construction des compétences de soin chez les étudiants.

Sans décrire en détail les différents programmes de formation qui ont été adaptés aux contraintes de chaque IFSI dans le cadre de cette recherche-action, il est possible de relever que chaque programme, quelle que soit sa durée, comportait des thèmes communs : une réflexion sur la notion de compétence et l'évaluation des compétences mais aussi des méthodes d'analyse de la tâche et d'analyse de l'activité. L'usage de ces méthodes à des fins pédagogiques pour la construction des compétences en soins infirmiers constituait un apport essentiel.

L'intervention alternait ainsi apports et entraînements en situation, pendant une durée de trois à huit jours de formation. Parfois, un sous-groupe de formateurs volontaires a connu un renforcement supplémentaire par rapport à leurs collègues. Cette équipe plus restreinte était, la plupart du temps, désignée pour mettre en œuvre la réforme dès la première rentrée des étudiants.

Notre point de départ est que l'analyse de la tâche n'est pas l'analyse de l'activité, puisque la tâche est d'abord ce qui doit être fait et que l'activité est ce que fait effectivement le sujet. L'écart entre la tâche et l'activité est donc systématique et la part créatrice du travail y réside souvent. Autant la tâche relève de l'anticipation et du programme d'action, l'activité est de l'ordre du vécu, du singulier, de l'émergeant, voire de l'indicible.

La tâche représente d'abord «un but à atteindre dans des conditions déterminées » ${ }^{(32)}$ et l'activité peut être comprise plutôt comme ce qui permet de faire face aux exigences d'une 
situation, c'est-à-dire ce qui relève de la confrontation d'un sujet et d'un contexte professionnel. Le travail est alors défini par « la confrontation des exigences de la tâche et les attitudes et séquences opérationnelles par lesquelles les individus observés répondent réellement à ces exigences » ${ }^{(33)}$.

La tâche renvoie au contexte de travail et aux prescriptions énoncées dans ce cadre contraint ${ }^{(34)}$. La prescription suppose l'activité du sujet mais ne la contient pas, il est nécessaire d'envisager au moins une tâche redéfinie par le sujet, laquelle va orienter son activité.

Avec les cadres formateurs, nous avons toujours envisagé le travail sous ses deux angles ${ }^{(35)}$. Du point de vue de la tâche et de la situation, il est nécessaire d'identifier ce qu'il faudrait savoir sur la situation mais aussi comment il faut agir pour produire une certaine efficacité. Un savoir, même pragmatique ne dit pas, à lui seul, comment se comporter en situation. Du point de vue du sujet et donc de son activité, il est utile de repérer les connaissances acquises et ses manières particulières, habituelles ou opportunistes, d'agir. Bien entendu, un professionnel infirmier connaîtra des difficultés à isoler rapidement et à coup sûr ses connaissances de ses manières d'agir : toutes participent également de l'efficacité soignante ; néanmoins les distinguer pour les besoins de l'analyse conduit à des opérations de comparaisons utiles pour la formation en soins infirmiers. Les formateurs peuvent, à l'aide d'outils appropriés, comparer les savoirs attendus, biomédicaux par exemple, avec les connaissances réelles des étudiants. De même, il est possible de comparer les manières d'agir attendues par les protocoles, les normes, les bonnes pratiques avec les manières d'agir effectives des étudiants. Les retours d'expériences, les simulations en «laboratoire de compétences » permettent cela. Les formateurs disposent alors d'un diagnostic leur permettant d'adapter leurs interventions aux besoins constatés des étudiants.

\section{Analyser les tâches : les situations-clés.}

Il existe de multiples façons d'analyser la tâche ${ }^{(36)}$, notamment à l'aide des documents prescripteurs ${ }^{(37)}$ ou en tant qu'artefacts symboliques « organisateurs de l'activité » ${ }^{(38)}$, nous avons retenu ici un type d'analyse qui privilégie la description des tâches à réaliser, les buts à poursuivre et les performances attendues, mais surtout une mise en perspective de ces éléments au regard des contextes, ce qui donne sens aux actes professionnels.

Trois entrées sont à considérer: le système de soins, les actions et les opérations. La tâche représente ici l'interface entre le système et les opérations concrètes attendues de chaque infirmier lorsqu'il doit agir dans ces situations.

Analyser une tâche consiste alors à repérer les trois niveaux, du plus large, celui du système de soins qui se comprend sous l'angle des besoins de santé à couvrir pour les populations, au plus profond, celui des opérations qui sont autant de tâches élémentaires.

Les tâches élémentaires sont toujours en relation avec un contexte, une situation particulière, les paramètres de la situation ne sont donc pas indifférents à la mise en œuvre des opérations et finalement à la réussite de l'action. D'autre part, l'efficacité d'une opération est liée au bon repérage de l'objet de l'intervention (surtout si l'objet est un sujet...) et à la maîtrise des méthodes, procédures et outils nécessaires.

Bien entendu, les tâches élémentaires ne se réalisent pas uniquement sous l'effet d'une «logique de situation » qui engagerait les acteurs à leurs dépens, ce que croient pourtant certains managers. Les savoirs, les buts et les raisons d'agir de l'infirmier en situation contribuent puissamment à orienter son activité, à sélectionner une tâche élémentaire plutôt qu'une autre, l'ordre dans lequel il va programmer les opérations ou l'attention qu'il va leur 
accorder. Dès lors, analyser une tâche prescrite consiste aussi à mettre en lumière les savoirs à maîtriser, les raisons d'agir conforme à l'état de l'art et les buts à poursuivre tels que les protocoles ou les bonnes pratiques les énoncent. Ce point de vue, qui consiste à former les étudiants à la connaissance fine des tâches est évidemment partagé par tous les formateurs, cela constitue d'ailleurs une grande partie de leur propre temps de travail. Reste à organiser l'information à délivrer aux étudiants en spécifiant ce qu'on peut attendre de l'analyse des tâches.

La figure 3 vise ainsi à outiller les cadres formateurs pour transmettre les situations-clés, présentées comme un pivot de la réforme de la formation en soins infirmiers. Ce type d'analyse est d'autant plus nécessaire que l'objectif est d'évaluer les compétences des étudiants. La situation-clé, ainsi comprise, se présentera comme un référentiel auquel on pourra comparer ce que les évaluateurs auront perçu de l'activité des étudiants. Comment les étudiants restituent-ils l'action dans un processus global de production de soins ? Poursuiventils des buts et des raisons d'agir suffisamment inscrits dans une culture et une pratique professionnelle raisonnée ? Comment s'acquittent-ils des opérations élémentaires, et pour quelle efficacité ?

Fig.3 Analyser une situation-clé

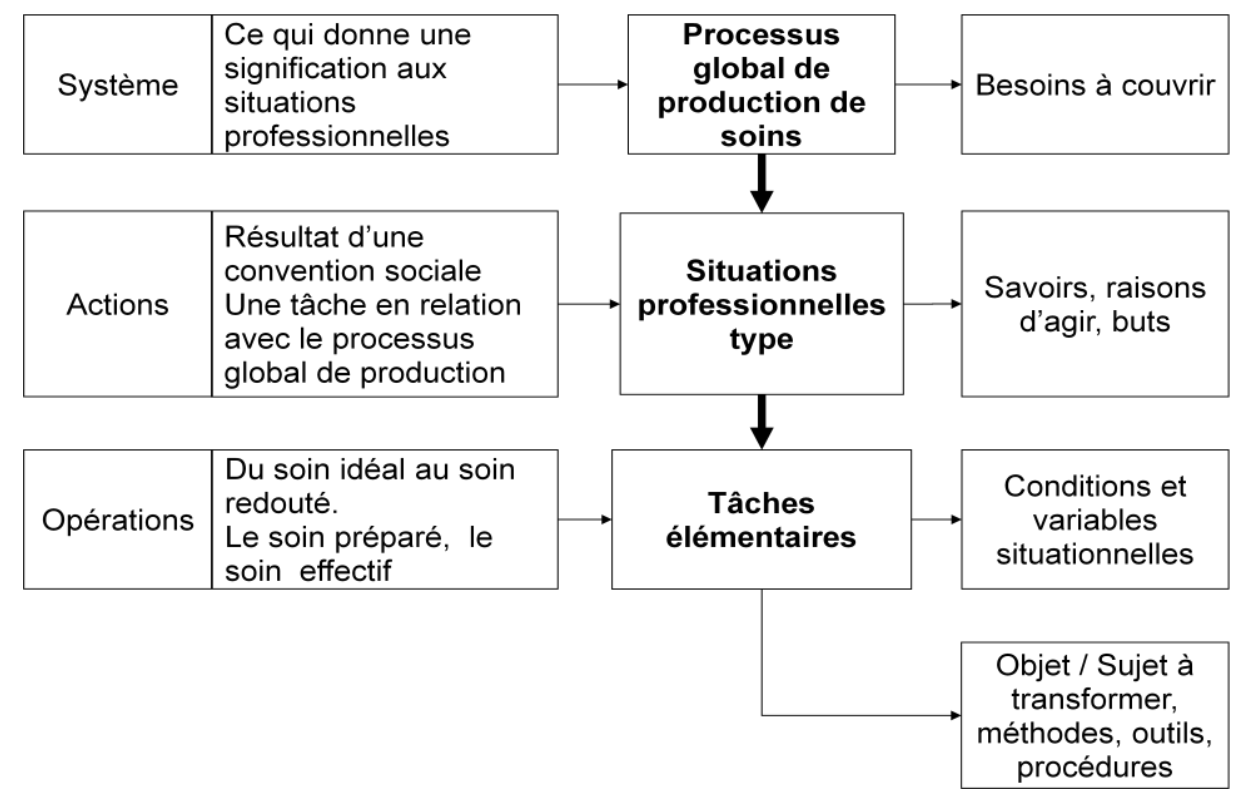

Chaque étudiant pourra commencer par sa propre auto-évaluation. L'exercice demande une certaine clairvoyance puisque l'exercice infirmier, aussi normé qu'il soit, produit d'abord un résultat «acceptable » et jamais tout-à-fait conforme à la norme, la marge d'appréciation de la conformité se juge à l'intérieur d'une enveloppe de moyens et de résultats. Il en irait des infirmiers comme des pilotes de chasse ${ }^{(39)}$, ils régulent leur action et gèrent des risquent dans le cadre d'une gestion par enveloppe : un soin technique, et un soin relationnel plus encore, sera accepté à l'intérieur de certaines limites, dans un intervalle entre le «soin idéal », celui qui se rencontre rarement et le «soin redouté » qui pourrait conduire à la mort du patient.

Ainsi, une situation-clé, une situation professionnelle type, telle que la pose d'une transfusion sanguine, peut être analysée selon cette méthode. Nous ne donnerons ici que quelques exemples des buts, tâches élémentaires ou autres conditions et variables situationnelles qu'il est possible de repérer. 
Dans le cas de la transfusion sanguine, le processus global de production de soins serait la préparation et la mise en œuvre des thérapeutiques selon les règles de sécurité, d'hygiène et d'asepsie. Les besoins de santé à couvrir tiennent essentiellement à la correction de l'anémie des patients.

Ici, le soin idéal est défini par une anémie du patient corrigée dans le respect de l'hygiène et de l'asepsie, avec une sécurité maximum dans la conformité aux protocoles et aucun effet secondaire causé par la transfusion. Quant au soin redouté, il résulterait d'une suite d'erreurs ou d'autres effets : erreur de patient ou erreur de poche de sang, erreur d'asepsie, impossibilité de trouver une voie veineuse, état de choc du patient lié à la transfusion, etc.

Pour la pose d'une transfusion sanguine, des savoirs sont requis et la liste ne saurait être exhaustive ni suffisamment précise : connaissances biomédicales de l'environnement sanguin et des paramètres biologiques, mécanismes physiopathologiques engendrés par la maladie, protocole de mise en œuvre de la transfusion sanguine, etc. Les raisons d'agir prennent en compte le pronostic vital après évaluation de la gravité de la situation et l'évolution des paramètres hémodynamiques du patient. Les buts qui doivent être poursuivis par les infirmiers sont notamment de contrôler la conformité de la prescription médicale et l'identitovigilance, d'informer le patient en lui apportant une attention soutenue, de respecter la procédure d'hémovigilance et la vérification ultime au lit du patient quitte à ce qu'une double vérification soit effectuée par un autre infirmier.

Les tâches élémentaires sont nombreuses : vérification de la traçabilité de la prescription médicale, de la fiabilité et de la pertinence des données ; identification du patient ; vérification de la compatibilité sanguine entre le patient et la poche de transfusion; pose de la transfusion en regard des critères d'hygiène et d'asepsie avec exactitude du débit (calcul de dose); surveillance des effets secondaires possibles liés à la pose de la transfusion sanguine.

Les conditions et variables situationnelles qui influencent les opérations sont, par exemple, celles de la situation d'urgence ou d'une situation régulée, de l'âge du patient, du type de pathologie, du lieu de la transfusion, à domicile ou en service de soins, etc.

L'objet ou le sujet à transformer est le système hémodynamique du patient. Les procédures à respecter sont celles de l'hémovigilance. Plusieurs outils sont à rassembler et à utiliser : tests de compatibilité, poches de sang et tubulures adéquates, régulateur de débit, antiseptique local, container pour recueillir l'aiguille et autres matériels, gants, éventuellement blouse, charlotte, masque, etc.

Chaque élément relevé dans cet exemple peut faire l'objet d'un enseignement spécifique, peut donner lieu à des tests de connaissance, pour lui-même ou, dans le cas d'une perspective plus globale, en relation avec le système sanitaire, les politiques hospitalières ou les sciences humaines et sociales.

\section{Analyser l'activité : un levier pour la professionnalisation des formateurs}

Si une situation de travail est rapidement analysable par les tâches à réaliser, les buts fixés et les performances attendues par la hiérarchie, les usagers, etc., l'activité du professionnel est, en revanche, décrite par les ressources mobilisées par l'individu pour agir dans des situations ordinaires de travail mais aussi ses stratégies d'adaptation pour faire face à l'imprévu.

Nous l'avons annoncé supra: dans le cadre de cette recherche-action en IFSI, nous limitons notre conception de la compétence à sa dimension cognitive. Nous avons donc invité les cadres formateurs à une approche des comportements efficaces, compétents, grâce aux clés de compréhension fournies par la psychologie cognitive et l'analyse de l'activité. 
Quelle a été cette «descente vers le cognitif », comme la caractérise Gérard VERGNAUD ? Comme nous l'avons décrit brièvement, nous avons initié les formateurs au concept de schème ${ }^{(6)}$ mais aussi à la théorie de la conceptualisation dans l'action ${ }^{(3)}$ et, en cas de besoin, à quelques repères sur l'analyse des activités de service ${ }^{(40,41)}$. L'outil d'analyse proposé aux cadres formateurs met ainsi l'accent sur les ressources cognitives qui se combinent dynamiquement pour agir avec efficacité.

L'une des premières ressources est celle qui consiste à comprendre la tâche, à en mesurer la portée et à prendre la décision de l'exécuter. Cette délibération interne au sujet sur la tâche aboutit à une redéfinition et à une première transformation de la tâche. Nous noterons d'ailleurs que la décision d'exécution est dépendante de l'auto-efficacité du professionnel, facteur qui intègre les processus motivationnels et volitionnels de contrôle de l'action. Une faible auto-efficacité conduira le soignant à éviter la tâche, à déployer des stratégies de protection, de contournement de la difficulté. A l'inverse, une forte auto-efficacité au travail est corrélée à la mise en action et à des probabilités de réussite, même si le sujet ne dispose pas de toutes les compétences avérées pour triompher de l'obstacle.

S'intéresser à la tâche redéfinie par le professionnel, c'est se donner une opportunité de comprendre comment et vers où le professionnel va orienter son action à venir, puisque de nombreuses causes d'échecs résident dans une mauvaise orientation de l'activité. Le sujet ne fait pas ce qu'il aurait du faire s'il avait correctement analysé la situation dans laquelle il se trouve placé, les tâches exigées et les méthodes à privilégier. Il se peut également que le professionnel compte sur ses routines, sur des comportements répétitifs mais souvent efficaces dans les cas habituels, sans chercher à s'adapter à la situation du moment.

Entrer par les buts s'apparente à dévider un fil d'Ariane pour se repérer dans l'organisation cognitive de l'activité d'un professionnel. Les buts que se fixe le professionnel (il est possible qu'il les exprime mais il est aussi possible de les inférer de sa pratique) se déclinent en sousbuts et en anticipations, ce qui « rend compte de l'intentionnalité de l'action » (42). Le professionnel contrôle son action en se donnant notamment des buts proximaux et distaux.

Les buts sont à analyser en relation avec les prises d'informations, les éléments essentiels à prendre en compte dans la situation, et les règles d'actions qui déclenchent la mise en œuvre effective.

L'activité comportant à la fois des éléments d'invariance et d'adaptation aux situations réelles de soin, il est aussi nécessaire de comprendre la hiérarchisation des buts et des sous-buts. Le cheminement d'un professionnel quand il passe de l'un à l'autre renseigne sur les compromis que le professionnel passe avec la situation, compromis dans sa relation au médecin, au patient, dans sa gestion immédiate des protocoles de soins. Accepte t-il les sollicitations des autres infirmiers, des aides-soignants alors qu'il se trouve au lit du patient? Quels compromis passe t-il avec les aspects relationnels de son exercice quand il lui faut revenir une fois de plus à la salle de soins, au bout du couloir, pour reprendre pansements et médicaments ?

Les buts et sous-buts sont emboités les uns dans les autres et l'infirmier change de buts quand l'un des sous-buts n'est pas atteint au niveau souhaité. Les changements de buts nous alertent sur les signes, souvent discrets, qui servent aux réajustements de l'action. Une organisation efficiente se cache souvent dans ces réajustements fréquents du déroulement de l'action.

La figure 4 vise à synthétiser l'ensemble des dimensions pertinentes pour analyser l'activité en soins infirmiers.

Fig. 4 Un outil pour analyser l'activité des étudiants en soins infirmiers 


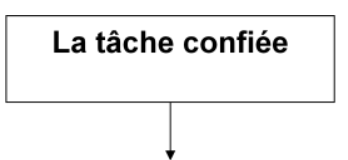

Quelle

commande (finalité et

enjeux de la

prescription)?

Quel(s) commanditaire

(s) ? Quels moyens

alloués pour la

réalisation?

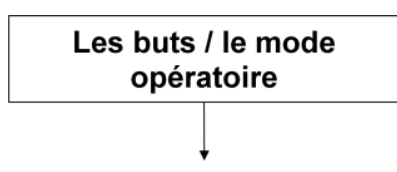

Quels buts généraux et quels buts intermédiaires? Quelles méthodes? Quelles étapes?

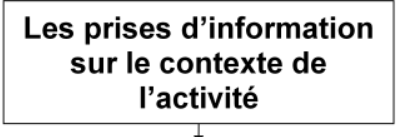

Les aspects essentiels de la situation professionnelle.

Quels sont les éléments les plus importants à prendre en compte pour réussir cette activité dans cette situation?

Ces informations étaient-elles disponibles?
Les réajustements en cours d'action I les difficultés rencontrées

Quels signes pour réajuster l'action en cours?

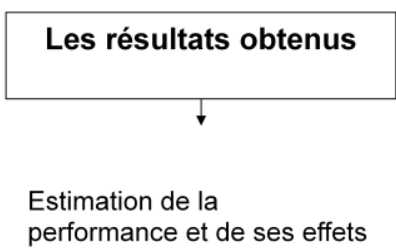

Tous les protagonistes de la formation, apprenants, formateurs, encadrants de stagiaires sur les terrains, sont concernés par les opérations d'analyse de la tâche ou d'analyse de l'activité même si les enjeux, les responsabilités ou les modes opératoires ne sont pas identiques. Il en sera de même pour l'évaluation des compétences.

\section{Former à l'évaluation des compétences}

Évaluer procède toujours d'une comparaison. Les performances d'un individu sont comparées à deux instants successifs ou les performances de deux individus sont comparées entre elles au même moment. De plus, les performances sont aussi comparées à un référentiel qui indique la nature de la performance à atteindre, voire son niveau et sa régularité.

La compétence est définie souvent comme une qualité attachée à l'individu qui agit efficacement, c'est-à-dire lorsqu'il obtient les performances attendues : " Des opérateurs capables de bonnes ou très bonnes performances sont considérés comme très compétents » ${ }^{(43)}$. Exprimée avec ce degré de généralité, cette relative évidence signifierait d'ailleurs qu'il est plus facile de reconnaître l'incompétence que la compétence. Une mauvaise performance est immédiatement identifiable par la majorité des personnes. Toutefois, l'efficacité immédiate de la performance ne suffit pas pour considérer que la compétence soit acquise par l'individu, le hasard peut aussi expliquer une performance à un moment donné ou dans des circonstances particulières. Il existe donc un décalage inévitable entre performance et compétence $^{(44)}$, voire, la compétence peut s'opposer à la performance : «la performance observée serait un indicateur plus ou moins fiable de la compétence [...]. Dans ce contexte, la compétence est une promesse de performance de tel niveau moyen $\gg{ }^{(45)}$.

Nous ne confondrons pas les notions de performance et de résultat. En effet, «définie conventionnellement, une performance est une réalisation : un résultat est ce qui en est issu. Autrement dit, un résultat est la conséquence d'une performance et non la performance ellemême » ${ }^{(5)}$.

La performance devient compétence (c'est-à-dire que l'individu fait l'objet d'un jugement social, d'une inférence de compétence à partir de l'observation d'une performance) 
lorsqu'elle respecte ces conditions d'efficacité, de reproductibilité et de régularité. La performance doit ainsi être reproductible et devrait se manifester de façon régulière.

Dans cette perspective sociocognitive, évaluer des compétences suppose de s'intéresser d'abord aux conditions d'apparition de la performance et à l'organisation cognitive de l'activité qu'elle sous-tend ${ }^{(7)}$ puis nous verrons comment l'évaluation de la compétence prend appui sur la manière dont l'individu régule son activité.

Premièrement, évaluer, c'est comparer si un individu sait faire plus de choses (par exemple, une toilette au lit ou au lavabo, un bilan sanguin, un pansement, la pose d'une sonde vésicale, la pose d'un cathéter, etc.) que lui-même en se comparant dans le temps, ou qu'un autre individu au même moment. C'est le résultat de l'activité qui est ici jugé. C'est utile mais réducteur et il faut aller plus loin.

Deuxièmement, si la réussite n'est pas une affaire de hasard, et qu'après plusieurs tests successifs, nous avons éliminé les réussites ou les échecs abusifs, il est instructif de comparer les façons de faire : le processus plutôt que le résultat. Un individu peut obtenir la même performance mais de manière plus efficiente, c'est-à-dire obtenir la même efficacité mais au moindre coût; cette efficience tellement attendue par les organisations hospitalières. Par exemple, l'infirmier qui aura rencontré son patient avant de rassembler le matériel pour un pansement ne se munira que du matériel nécessaire, sans superflu, et que du matériel adapté à la prescription du chirurgien, avant de rentrer dans la chambre. A l'inverse, un infirmier plus novice pourrait prendre du matériel lui permettant de faire face à plusieurs cas de figure, or tout matériel rentrant dans une chambre doit être éliminé à l'issue du soin. Le soin est le même, l'efficience varie. Il est aussi possible de comprendre la «meilleure manière » par d'autres critères : rapidité, fiabilité, etc.

Troisièmement, à performance égale, l'individu peut disposer d'un répertoire de stratégies plus étendu. Les situations rencontrées ne sont jamais identiques et à l'intérieur d'une même classe de situations, la compétence n'est pas reconnue seulement dans des situations similaires mais aussi dans des classes de situations élargies où la dose d'imprévu augmente. Il est souhaitable de s'adapter et de traiter des cas nouveaux. Il est toujours indispensable de choisir la bonne procédure mais il est aussi parfois nécessaire de passer d'une procédure à une autre. La qualité du diagnostic infirmier et du raisonnement clinique permettent de comprendre pourquoi deux individus confrontés au même problème peuvent obtenir la même efficacité de soins en employant pourtant deux méthodes différentes. Par exemple, un infirmier qui doit réaliser une toilette chez une personne âgée incontinente voit deux possibilités s'offrir à lui : soit la réalisation de la toilette selon la logique du soin du plus propre au plus sale, du visage aux pieds; soit, son raisonnement professionnel lui permet d'évaluer l'état de la personne soignée (confort, bien-être, etc.). Si la personne est souillée, la logique du soin cède devant la priorité qui sera alors de nettoyer le siège avant de revenir à une prise en charge « classique », du plus propre au plus sale.

Quatrièmement, la compétence, et nous parlerons alors d'expertise, se manifeste dans des situations tout à fait nouvelles où l'individu doit mobiliser ses ressources pour faire face à une question inédite. L'infirmier expert n'est pas celui qui a reproduit un grand nombre de fois le même geste technique ou a suivi le même raisonnement clinique, il est plutôt en mesure de traiter des problèmes nouveaux qu'il n'a jamais rencontrés auparavant. Il sait mobiliser, agréger et combiner des ressources supplémentaires dans ce but. L'improvisation face aux difficultés compte alors plus que la routine. Par exemple, lorsqu'un infirmier se retrouve seul à poser une sonde vésicale, soin qui requiert normalement la présence de deux personnes, il prend des libertés avec le protocole ce qui se traduit par une préparation plus minutieuse encore du matériel, par l'ouverture peut-être plus précoce de dispositifs stériles, par une anticipation des gestes et par la création de conditions favorables chez la personne soignée. L'improvisation réside ici dans la préparation du soin et dans la planification de la réalisation 
hors protocole. Tester la débrouillardise de l'infirmer est aussi une manière d'évaluer la compétence. Dans bien des cas, l'improvisation peut être testée en laboratoire de compétence, sans les risques des situations réelles au lit du malade, ce qui renseigne sur le type d'organisation cognitive de l'activité de tel ou tel étudiant en soins infirmiers.

Évaluer, c'est donc comparer, mais c'est aussi se donner une opportunité de régulation des apprentissages en formation. Nous pouvons comparer des buts, ceux qui sont prescrits par l'organisation hospitalière aux buts effectivement poursuivis par l'infirmier; des résultats attendus aux performances constatées; des stratégies cognitives conduisant aux pratiques recommandées aux stratégies cognitives qui sont constatées dans l'action concrète. L'évaluation possède néanmoins une deuxième fonction, celle de réguler les apprentissages ${ }^{(46)}$. Elle vise à traduire les écarts constatés en action correctrices et tenter de réduire ces écarts pour atteindre les buts fixés et obtenir l'efficacité prévue.

Lors de la recherche-action, un outil a été transmis aux cadres formateurs: la «boucle comparaison / régulation », inspirée de Jacques LEPLAT et adaptée à la formation en soins infirmiers.

Le principe de cette boucle comparaison/régulation est de reconstituer l'ensemble dynamique de l'activité, à travers son analyse, et l'interdépendance avec les tâches prescrites, mais aussi les opérations de comparaison à finalité d'évaluation des compétences acquises et la source des actions correctrices, c'est-à-dire les actions des formateurs sur les apprenants et, plus sûrement encore, sur les milieux d'apprentissage.

L'acquisition de compétence est un processus, il est initié de façon explicite par les cadres formateurs et il fait l'objet d'une évaluation constante en appui sur des référentiels externes aux apprenants et aux cadres formateurs. En cela, la boucle comparaison/ régulation est largement ouverte sur l'environnement professionnel dont elle intègre les principales composantes normatives.

Dans le détail, cette boucle est composée de trois processus articulés les uns aux autres. Le premier processus de comparaison traite les informations qui viennent d'être mentionnées lors des opérations d'évaluation des compétences : réussir de nombreuses actions différentes et avec la bonne manière, disposer de stratégies mobilisables face aux problèmes connus ou imprévus. La comparaison s'établit entre des référentiels externes (ils peuvent bien entendu être appropriés par les individus pour servir leur auto-évaluation) et des constats portant sur les performances et les modes de réalisation des actions.

L'écart éventuellement constaté entre ce qui est attendu et ce qui observé est traité par le second processus, celui de régulation des apprentissages. Nous sommes ici au cœur de la didactique professionnelle avec la construction de milieux d'apprentissage ${ }^{(47)}$, les conditions du développement professionnel ${ }^{(40)}$ et une ingénierie de la compétence prenant appui sur des régulations «en boucle courte » ou en «boucle longue » ${ }^{(48)}$.

Les actions régulatrices ou correctrices sont mises en œuvre soit par le formateur, soit par l'infirmier qui encadre le stagiaire dans le service de soins, soit par l'apprenant lui-même dans une visée autoformatrice. S'enclenche alors le troisième processus de construction de compétence. Celui-ci est conditionné par le développement d'invariants opératoires et par la conceptualisation dans et pour l'action. L'action est organisée et cette organisation repose sur un certain nombre d'éléments invariants qui permettent l'adaptation à la diversité des situations ${ }^{(1)}$.

La figure 5 modélise l'ensemble des éléments constitutifs de ces processus et de leurs interactions.

\section{Fig. 5 La boucle comparaisons / régulation}




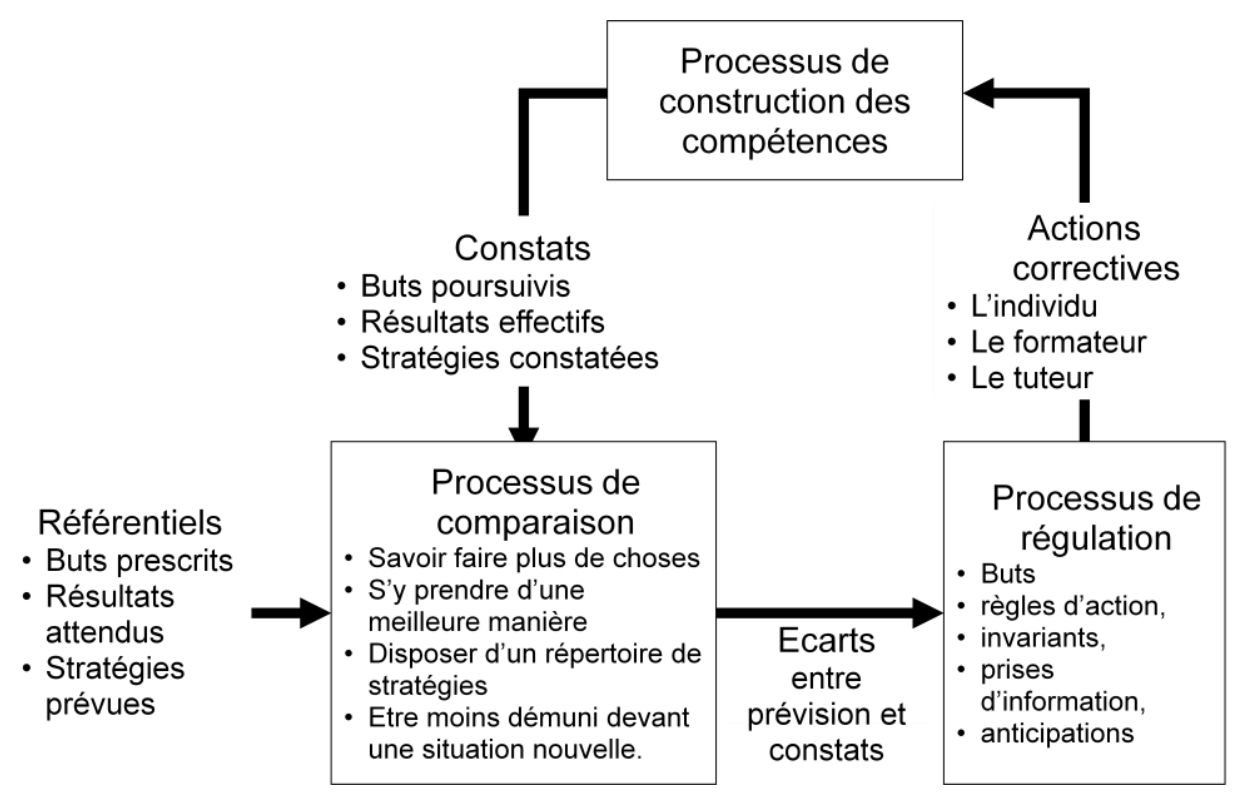

Avec ce modèle de l'évaluation des compétences, nous ne dissocions pas l'évaluation de la régulation et du développement de compétences. En tout état de cause, la compétence met en jeu plusieurs niveaux, celui de la performance mais aussi celui de la complexité de la situation de travail et également celui du niveau de risques de l'action. Il nous faut effectivement penser ces niveaux en interaction constante.

Dans ce long chapitre dédié à la méthodologie, nous avons voulu expliquer les bases de notre intervention lors de cette recherche-action. Pour comprendre la relation qui s'établit entre analyse de l'activité et auto-efficacité collective, il fallait en comprendre les termes respectifs. Nous allons maintenant exposer les résultats et les analyser.

\section{Une augmentation des scores d'auto-efficacité}

En introduction de cette partie, nous rappellerons l'intérêt porté aux effets de l'auto-efficacité individuelle et collective. Une forte auto-efficacité est corrélée aux performances, une fois les autres facteurs contrôlés, mais une forte auto-efficacité permet aussi de prédire une plus grande persévérance et une meilleure résilience face aux obstacles ainsi qu'une autorégulation de l'activité plus efficace.

Nous nous interrogeons ici sur la construction de l'auto-efficacité collective en lien avec l'analyse de l'activité et, plus généralement, de l'analyse du travail infirmier que pourraient conduite des cadres formateurs en IFSI.

\section{Une meilleure efficacité collective perçue}

Les données qualitatives sur l'auto-efficacité ont été collectées à l'occasion de temps formels tels que des moments de libre expression dans le groupe au démarrage ou à la fin des sessions collectives et donc en présence de la totalité du groupe. Il est à noter que les énoncés positifs ont recueilli l'assentiment général et que les énoncés négatifs ont parfois été vigoureusement discutés, essentiellement à la fin de l'intervention. 
Plusieurs paramètres ont été enregistrés : le partage des croyances quant à la capacité du groupe à faire face à la réforme et à l'approche par compétences promue par le ministère de tutelle, la coordination du groupe pour unir leurs efforts, concevoir et réaliser une ingénierie pédagogique efficiente et orientée vers l'approche par compétences, l'anticipation des ressources à mobiliser collectivement.

Pour les cinq IFSI et leurs 109 formateurs, un nombre identique d'énoncés a pu être recueilli : "Que diriez-vous, en une phrase, de la capacité de votre équipe de formateurs à mettre en œuvre une approche par les compétences en soins infirmiers ?». Ils ont été classés selon leur nature : indicateurs motivationnels tels que l'engagement, construits motivationnels, facteurs individuels et situationnels, effectivité des réalisations pédagogiques à destination des étudiants et estimation de la performance du groupe à conduire la réforme, mais aussi selon leur orientation: positive ou négative quant à la possibilité effective de mise en œuvre collective. Les tableaux 3 et 4 exposent les résultats principaux.

Tableau 3 Prétest - Appréciations de l'auto-efficacité collective à la mise en œuvre de la réforme

\begin{tabular}{|c|c|c|c|c|}
\hline $\begin{array}{l}\text { IFSI (nombre } \\
\text { de cadres } \\
\text { formateurs) }\end{array}$ & $\begin{array}{l}\text { Énoncés } \\
\text { orientés } \\
\text { positivement }\end{array}$ & Exemple & $\begin{array}{l}\text { Énoncés } \\
\text { orientés } \\
\text { négativement }\end{array}$ & Exemple \\
\hline $\mathrm{A}(\mathrm{n}=15)$ & 4 & $\begin{array}{l}\text { Des collègues ont travaillé } \\
\text { dans des groupes régionaux, } \\
\text { ils ont des bonnes idées. }\end{array}$ & 11 & $\begin{array}{l}\text { Nous n'avons pas les moyens } \\
\text { d'y arriver, nous ne sommes } \\
\text { pas prêts. }\end{array}$ \\
\hline$B(n=20)$ & 15 & $\begin{array}{l}\text { S'il le faut, on appliquera sans } \\
\text { se poser trop de questions. }\end{array}$ & 5 & $\begin{array}{l}\text { On ne nous a rien expliqué } \\
\text { jusqu'à présent, tout est à } \\
\text { faire. }\end{array}$ \\
\hline$C(n=30)$ & 24 & $\begin{array}{l}\text { On a quelques bases et } \\
\text { quelques uns voudraient } \\
\text { expérimenter les labos de } \\
\text { compétence. }\end{array}$ & 6 & $\begin{array}{l}\text { Ca n'a pas vraiment marché } \\
\text { avec les AS [les formations } \\
\text { d'aides-soignantes]. }\end{array}$ \\
\hline $\mathrm{D}(\mathrm{n}=20)$ & 18 & $\begin{array}{l}\text { Nous avons déjà des pratiques } \\
\text { de formation qui vont dans ce } \\
\text { sens. }\end{array}$ & 2 & $\begin{array}{l}\text { Ca va ramer dans les } \\
\text { équipes, on n'est pas tous au } \\
\text { même niveau. }\end{array}$ \\
\hline$E(n=24)$ & 18 & $\begin{array}{l}\text { Ca nous fera sortir de la } \\
\text { routine, on en a besoin. }\end{array}$ & 6 & $\begin{array}{l}\text { Je plains les étudiants qui } \\
\text { vont essuyer les plâtres et } \\
\text { nous avec... }\end{array}$ \\
\hline $\begin{array}{l}\text { Ensemble } \\
(\mathrm{n}=109)\end{array}$ & $79(72 \%)$ & & $30(28 \%)$ & \\
\hline
\end{tabular}

Tableau 4 Posttest - Appréciations de l'auto-efficacité collective à la mise en œuvre de la réforme

\begin{tabular}{|l|c|l|l|l|}
\hline $\begin{array}{l}\text { IFSI (nombre } \\
\text { de cadres } \\
\text { formateurs })\end{array}$ & $\begin{array}{l}\text { Énoncés } \\
\text { orientés } \\
\text { positivement }\end{array}$ & Exemple & $\begin{array}{l}\text { Énoncés } \\
\text { orientés } \\
\text { négativement }\end{array}$ & Exemple \\
\hline $\mathrm{A}(\mathrm{n}=15)$ & 12 & $\begin{array}{l}\text { C'était l'évaluation des } \\
\text { compétences qui nous } \\
\text { paniquaient. Maintenant, on y } \\
\text { voit plus clair. Ouf ! }\end{array}$ & 3 & $\begin{array}{l}\text { Ca sera difficile de tout gérer } \\
\text { à la fois. Il faudra bien } \\
\text { continuer avec les promos en } \\
\text { cours. }\end{array}$ \\
\hline $\mathrm{B}(\mathrm{n}=20)$ & 15 & $\begin{array}{l}\text { Après cette formation, on } \\
\text { voudrait encore renforcer nos } \\
\text { premières expérimentations. }\end{array}$ & 5 & $\begin{array}{l}\text { On ne sera pas tous sur le } \\
\text { même rythme. Quid des } \\
\text { résistances au changement? }\end{array}$ \\
\hline $\mathrm{C}(\mathrm{n}=30)$ & 26 & $\begin{array}{l}\text { Il nous reste à convaincre nos } \\
\text { collègues sur les terrains [dans } \\
\text { les services de soins]. }\end{array}$ & 4 & $\begin{array}{l}\text { Les habitudes des étudiants } \\
\text { vont être chamboulées. Est- } \\
\text { ce qu'ils accepteront de nous } \\
\text { suivre ? Ce n'est pas certain. }\end{array}$ \\
\hline $\mathrm{D}(\mathrm{n}=20)$ & 17 & Je crois que nous avons & 3 & Le portfolio [livret \\
\hline
\end{tabular}




\begin{tabular}{|l|l|l|l|l|}
\hline & & $\begin{array}{l}\text { compris que ce que nous } \\
\text { pouvions faire de nos vieilles } \\
\text { vidéos pour développer les } \\
\text { compétences. }\end{array}$ & $\begin{array}{l}\text { d'évaluation des } \\
\text { compétences des étudiants en } \\
\text { cours et en fin de formation } \\
\text { qui est imposé par la } \\
\text { réforme] est trop complexe. } \\
\text { On ne voit pas comment on } \\
\text { pourra certifier des } \\
\text { compétences. }\end{array}$ \\
\hline $\mathrm{E}(\mathrm{n}=24)$ & 20 & $\begin{array}{l}\text { L'analyse de l'activité, c'est } \\
\text { du concret. On va pouvoir } \\
\text { s'appuyer sur l'expérience } \\
\text { quotidienne. }\end{array}$ & 4 & $\begin{array}{l}\text { On arrivera toujours à } \\
\text { recycler nos cours. }\end{array}$ \\
\hline $\begin{array}{l}\text { Ensemble } \\
(\mathrm{n}=109)\end{array}$ & $90(83 \%)$ & & $19(17 \%)$ & \\
\hline
\end{tabular}

Si l'on en juge par le nombre d'énoncés positifs qui augmente globalement, de $72 \%$ à $83 \%$, sur l'ensemble des IFSI et celui des énoncés négatifs qui diminue d'autant, l'auto-efficacité collective s'améliore. Il est à noter tout de même que l'IFSI B n'a pas connu d'évolution et que le nombre d'énoncés positifs décroit d'un point pour l'IFSI D. La progression n'est donc pas constante pour les cinq IFSI.

Les énoncés peuvent parfois changer de nature entre le prétest et le posttest.

Les énoncés positifs prétest portent majoritairement sur des ressources supposées être partagées par le collectif, celles qui permettront de se préparer à la réforme, ou sur des qualités d'engagement ou d'innovation jugées intrinsèques à l'équipe. Posttest, l'autoefficacité collective s'appuie plutôt sur des ressources liées à la didactique professionnelle.

Les premiers énoncés négatifs mettent l'accent sur des contraintes extérieures qui empêchent la mobilisation, des expériences mal vécues ou des manques de cohérence présumés de l'équipe. Posttest, ces obstacles demeurent et se conjuguent avec des contraintes externes : étudiants jugés peu matures, arrêté de formation catalogué comme «mal conçu», organisation perçue comme peu performante.

Le niveau d'auto-efficacité collective augmente après l'intervention, les premières réalisations en cours d'intervention entretiennent la croyance des formateurs dans leur capacité à réussir collectivement.

Les effets de l'initiation à l'analyse du travail dans le cadre de la formation des compétences cliniques, relationnelles et organisationnelles des infirmiers sont d'autant plus reconnus que des sous-groupes de formateurs, porteurs des premières réalisations, ont été constitués et ont suivi un programme renforcé. Lorsque ces sous-groupes ont été réunis, nous avons pu observer une augmentation de l'interdépendance entre les formateurs et un besoin de coordination plus fort. Or, plus les membres d'un groupe sont interdépendants, plus la valeur prédictive de l'auto-efficacité collective sur les comportements est bonne.

Les interventions ont été réalisées avec des équipes qui n'avaient pas été formées auparavant à la didactique professionnelle. De plus, rares sont les formateurs experts en pédagogie qui ont suivi une formation universitaire de haut niveau, néanmoins, le sentiment de compétence collective augmente après l'intervention. Ce résultat semble montrer que l'analyse de l'activité est un facteur d'auto-efficacité au travail, individuelle et collective.

\section{Des scores d'auto-efficacité individuelle et collective qui augmentent}


L'auto-efficacité collective a été mesurée avant et après l'intervention de sensibilisation à la didactique professionnelle. Le score de chaque équipe a été déterminé par la moyenne des scores individuels. Les tableaux 5 et 6 rendent compte de ces mesures.

Tableau 5 Prétest - Mesure de la tendance centrale de l'auto-efficacité collective

\begin{tabular}{|l|c|c|c|c|c|c|}
\hline $\begin{array}{l}\text { IFSI (nombre } \\
\text { de cadres } \\
\text { formateurs) }\end{array}$ & Score mini & Score maxi & Score moyen & Mode & Score médian & Écart-type \\
\hline A (n=15) & 17 & 35 & 27,13 & 28 & 28 & 4,91 \\
\hline B (n=20) & 24 & 40 & 30,20 & 30 & 30 & 3,91 \\
\hline C $(n=30)$ & 20 & 40 & 32,00 & 32 & 32 & 4,68 \\
\hline $\mathrm{D}(\mathrm{n}=20)$ & 25 & 37 & 31,00 & 32 & 32 & 3,81 \\
\hline $\mathrm{E}(\mathrm{n}=24)$ & 17 & 40 & 28,75 & 29 & 29 & 5,80 \\
\hline $\begin{array}{l}\text { Ensemble } \\
(\mathrm{n}=109)\end{array}$ & 17 & 40 & 30,10 & 32 & 30 & 4,92 \\
\hline
\end{tabular}

Tableau 6 Posttest - Mesure de la tendance centrale de l'auto-efficacité collective

\begin{tabular}{|l|c|c|c|c|c|c|}
\hline $\begin{array}{l}\text { IFSI (nombre } \\
\text { de cadres } \\
\text { formateurs) }\end{array}$ & Score mini & Score maxi & Score moyen & Mode & Score médian & Écart-type \\
\hline A (n=15) & 25 & 35 & 30,93 & 32 & 32 & 3,17 \\
\hline B $(\mathrm{n}=20)$ & 25 & 35 & 31,25 & 32 & 32 & 2,83 \\
\hline $\mathrm{C}(\mathrm{n}=30)$ & 25 & 40 & 33,47 & 32 & 33 & 3,96 \\
\hline $\mathrm{D}(\mathrm{n}=20)$ & 19 & 40 & 32,65 & 32 & 33 & 5,49 \\
\hline $\mathrm{E}(\mathrm{n}=24)$ & 25 & 40 & 30,96 & 31 & 31 & 3,85 \\
\hline $\begin{array}{l}\text { Ensemble } \\
(\mathrm{n}=109)\end{array}$ & 19 & 40 & 32,01 & 32 & 32 & 4,07 \\
\hline
\end{tabular}

Sur une échelle de 10 à 40 points, le score minimum augmente de deux points et le score maximum est tout de suite atteint avec 40 points.

D’une manière générale, les modes, moyennes et scores médians sont groupés mais leurs valeurs peuvent sensiblement différer selon les IFSI et le moment de la mesure. Modes et scores médians accompagnent l'évolution de la moyenne, c'est-à-dire de l'auto-efficacité collective. Chaque IFSI a vu augmenter son score d'auto-efficacité collective.

En matière d'auto-efficacité collective, la mesure de l'écart-type renseigne sur la cohérence du groupe dans les réponses individuelles. Un écart-type faible, à moyenne d'auto-efficacité égale, laisse présager d'une auto-efficacité collective plus consistante. Chaque écart-type a diminué après l'intervention.

Il est à noter que les IFSI A et E dont les scores étaient les plus bas et inférieurs à 30 points ont connu l'augmentation la plus importante, voir le tableau 7.

Tableau 7 Évolution des scores d'auto-efficacité collective-Prétest, posttest

\begin{tabular}{|l|c|c|c|}
\hline $\begin{array}{l}\text { IFSI (nombre de cadres } \\
\text { formateurs) }\end{array}$ & Score moyen initial & Score moyen final & Évolution \\
\hline A $(n=15)$ & 27,13 & 30,93 & $+3,08$ \\
\hline B $(n=20)$ & 30,20 & 31,25 & $+1,05$ \\
\hline C $(n=30)$ & 32,00 & 33,47 & $+1,47$ \\
\hline$D(n=20)$ & 31,00 & 32,65 & $+1,65$ \\
\hline
\end{tabular}




\begin{tabular}{|l|l|l|l|}
\hline $\mathrm{E}(\mathrm{n}=24)$ & 28,75 & 30,96 & $+2,21$ \\
\hline Ensemble $(\mathrm{n}=109)$ & 30,10 & 32,01 & $+1,91$ \\
\hline
\end{tabular}

La valeur de 30 points représente une valeur de référence avec cette échelle, tant pour l'autoefficacité individuelle ${ }^{(27)}$ que pour l'auto-efficacité collective au travail ${ }^{(28)}$ : «L'ensemble de la population analysée présente un sentiment d'efficacité personnelle élevé avec une moyenne de 29.94 sur 40.00 ainsi qu'un sentiment d'efficacité collective élevé avec une moyenne de 29.67 sur 40.00 ». Il en est de même pour l'auto-efficacité mesurée avec des échelles plus générales (49) pour 17000 personnes. Ainsi, nous pouvons considérer qu'une forte autoefficacité est établie au-delà de 30 points.

Pourquoi avons-nous constaté une augmentation de l'auto-efficacité collective suite à ces phases d'analyse de l'activité ? Il semblerait que la formation à l'analyse de l'activité pour ces cadres formateurs représente un facteur de construction de l'auto-efficacité collective parce qu'elle contribue à générer un triple processus, au-delà même de l'augmentation des connaissances sur l'analyse du travail.

Le premier est un processus de généralisation et de reconstruction de sens. Il intègre les expériences antérieures de pédagogie active, d'approche par problèmes, voire des tentatives de simulation en laboratoire, mais la didactique professionnelle donne un surcroit de sens, par l'étayage théorique, à ces pratiques qui apparaissent finalement très empiriques aux participants eux-mêmes.

Le deuxième augmente le niveau d'abstraction dans la compréhension de ses comportements de formateurs et dans les logiques d'apprenance. Or, les cadres formateurs de ces IFSI étaient majoritairement à la recherche de renouvellement des pratiques et surtout d'une plus grande cohérence et solidarité d'équipe face à la réforme annoncée. Réinterroger leurs pratiques en les réinterprétant à la lumière d'une ingénierie des compétences a favorisé ce processus d'abstraction.

Enfin, l'analyse des expérimentations qui ont été réalisées spontanément en cours d'intervention, le travail autour des cas concrets, les échanges dans le groupe qui ont permis de se constituer une culture commune autour des compétences et de la didactique professionnelle, tout cet ensemble a produit un effet d'attribution des actes et des résultats au groupe, et à soi-même dans le groupe. Parce que l'efficacité collective représente un élément de la structure sociale de l'équipe de formateurs, les premiers succès sont analysés comme le reflet de la compétence perçue du groupe et comme une promesse de succès à l'avenir sans que les échecs ne soient attribués directement au groupe.

Ces trois processus observés en cours des interventions sont vraisemblablement des facteurs du développement de l'auto-efficacité collective appliquée à la formation en soins infirmiers.

\section{Les limites de cette étude}

Si cette étude a le mérite de produire des données peu fréquentes sur l'auto-efficacité des formateurs, elle trouve sa limite dans une certaine forme de recherche-action qui conduit à privilégier l'intervention sur des terrains déjà repérés et sensibles tout en délaissant des terrains plus éloignés (des IFSI qui ont peu anticipé la réforme) mais certainement tout aussi représentatifs du secteur.

Une autre limite réside dans la difficulté à mesurer l'auto-efficacité collective et des dispositifs empiriques qui ne sont jamais tout-à-fait satisfaisants. 
Il serait également nécessaire d'introduire une perspective longitudinale et de s'intéresser aux phases de construction de l'auto-efficacité collective, mais aussi de déclin et de reconstruction pour mieux interpréter les résultats obtenus à un moment donné, épisode tout à fait particulier dans cette histoire de la formation aux soins infirmiers.

Enfin, nous noterons l'absence d'un groupe témoin (plan prétest-posttest à groupe unique) qui aurait permis de tester plusieurs hypothèses concurrentes.

Pour autant, un certain nombre de précautions ont été prises pour contrôler les effets de la mesure répétée. La mesure intervient dès que possible à la fin de l'intervention pour limiter la portée des autres situations qui pourraient agir sur la construction de l'auto-efficacité individuelle et collective. Nous sommes en conditions naturelles et non en conditions de laboratoire, la vie quotidienne et professionnelle ne s'arrête pas pendant le temps de l'intervention. Notamment, les réunions d'équipes formelles ou informelles, sur le sujet de l'application de la réforme se poursuivent, des journées professionnelles se tiennent auxquelles participent volontiers les formateurs et les directeurs des soins, le ministère accélère son travail de conception et de concertation pour rédiger un arrêté de formation dont la publication est prévue à l'été 2009. De fait, nous ne pouvons pas mesurer avec certitude l'influence que ces événements ont eu sur la construction de l'auto-efficacité, s'ils ont favorisé ou entravé son développement et avec quelle force.

\section{Conclusion}

Lorsqu'à l'issue de l'intervention, nous avons interrogé quelques cadres formateurs ou directeurs d'instituts, ils nous ont rapporté quelques changements observés.

Les formateurs se disent plus persévérants et plus engagés dans la réforme proposée par le ministère de la santé. Leur résilience s'est accrue face aux difficultés de conception d'un dispositif de formation orienté vers les compétences.

Les signes d'une augmentation de l'auto-efficacité collective sont perceptibles et nous pouvons attendre que ces équipes se fixent des objectifs plus élevés. Collectivement, ces équipes devraient mieux coordonner leurs efforts et décider de manière plus convergente des réajustements à apporter au démarrage du nouveau plan de formation, ces prochains mois.

Aux directeurs des soins qui dirigent ces établissements de prendre le relai aujourd'hui. Les objectifs de management, de gestion du changement, sont là, présents devant eux : «La réussite de l'efficacité collective nécessite des méthodes convaincantes réunissant les intérêts partisans dans un projet commun. Les projets unificateurs doivent être explicites et accessibles grâce à un effort concerté ${ }^{(5)}$. Nous leur souhaitons pleine réussite.

Bibliographie

1. PASTRÉ P., MAYEN P., VERGNAUD G. La didactique professionnelle. Revue française de pédagogie. 2006;154.

2. MERCHIERS J. A-t-on besoin de compétence pour travailler ? Travailler 2000(4).

3. PASTRÉ P. Champs conceptuels et champs professionnels. In: MERRI M., editor. Activité humaine et conceptualisation Questions à Gérard Vergnaud. Toulouse: Presses universitaires du Mirail; 2007. 
4. PY J., SOMAT A. Normativité, conformité et clairvoyance : leurs effets sur le jugement évaluatif. In: BEAUVOIS J-L., JOULE, MONTEIL J-M., editor. Perspectives cognitives et conduites sociales. Fribourg: Delval; 1991.

5. BANDURA A. Auto-efficacité. Le sentiment d'efficacité personnelle. Bruxelles: De Boeck; 2003.

6. VERGNAUD G. La théorie des champs conceptuels. Recherches en didactique des mathématiques. 1990;10 (2/3).

7. VERGNAUD G. Une approche cognitive des compétences et de l'activité. In:

Université de Bretagne, editor. Compétences, emploi et enseignement supérieur

L'enseignement supérieur prépare-t-il à l'emploi ? Rennes; 2006.

8. VERGNAUD G. Le développement cognitif de l'adulte. In: CARRÉ P., CASPAR P., editor. Traité des sciences et des techniques de la formation. Paris: Dunod; 1999.

9. BANDURA A. Social cognitive theory : an agentic perspective. Annual review of psychology. 2001;52:1 - 26.

10. CARRÉ P. Bandura : une psychologie pour le XXIe siècle ? Savoirs : revue internationale de recherche en éducation et formation des adultes. 2004; $\mathrm{N}^{\circ}$ Hors-série Autour de l'œuvre de Bandura.

11. ZIMMERMAN B. Efficacité perçue et autorégulation des apprentissages durant les études : une vision cyclique. In: CARRÉ P., MOISAN A., editor. La formation autodirigée Aspects psychologiques et pédagogiques. Paris: L'Harmattan; 2002.

12. SIMON L., TARDIF N. Le sentiment d'efficacité personnelle à la base du développement des compétences. Le point en administration scolaire. 2006;9 (1).

13. LINDSEY D., MATHIEU J., HEFFNER T., BRASS D. Team efficacy, potency, and performance: A longitudinal examination of reciprocal processes. Annual Meeting of the Society for Industrial and Organizational Psychology,. Nashville; 1994.

14. ZACCARO S., BLAIR V., PETERSON C., ZAZANIS M. Collective efficacy. In: MADDUX J., editor. Self-efficacy, adaptation, and adjustment Theory, research, and application New York: Plenum Press; 1995.

15. SHAMIR B. Calculations, values, and identities: The resources of collectivistic work motivation. Human Relations. 1990;43.

16. GIST M. Self-efficacy: implications for organizational behavior and human resource management. Academy of Management Review. 1987;12.

17. SPINK K.S. Collective efficacy in the sport setting. International Journal of Sport Psychology. 1990;21 (Special Issue: The group in sport and physical activity).

18. TROLLAT A-F. Vers l'autoformation à l'école : nécessité d'un travail en équipe des enseignants et sentiment d'efficacité collective. In: AECSE, editor. Congrès AECSE Actualité de la Recherche en Education et en Formation. Strasbourg; 2007.

19. CAPRARA G., BARBARANELli C., BORGOGNI L., PETITTA L., RUBINACCI

A. Teachers', school staff's and parents' efficacy beliefs as determinants of attitudes toward school. European journal of psychology of education. 2003;18(1).

20. LAHMOUZ K., DUYCK J-Y. Le stress professionnel, l'implication organisationnelle et l'auto-efficacité : cas d'une restructuration - les apports d'une étude quantitative. Actes du congrès de l'AGRH. Dakar; 2008.

21. CHOUANIERE D. Stress et risques psychosociaux : concepts et prévention.

Documents pour le médecin du travail. 2006(106).

22. JASSERON C., ESTRYN-BEHAR M., LE NEZET O., RAHHALI I. Les facteurs liés à l'abandon prématuré de la profession soignante. Confirmation de leur impact à un an d'intervalle. Analyse longitudinale des soignants de l'enquête PRESST/NEXT. Recherches en soins infirmiers. 2006(85). 
23. ESTRYN-BÉHAR M. Santé et satisfaction des soignants au travail en France et en Europe. Rennes: Presses de l'EHESP; 2008.

24. SELIGMAN M. Helplessness : On depression, development, and death. San Francisco: W. H. Freeman; 1975.

25. SAVOYANT A. La professionnalisation des personnels infirmiers. Marseille: EQUAL - TRANSFERS; 2005.

26. BARTH B.-M. Construire son savoir, L'adulte en formation. Bruxelles: De Boeck Université; 1996.

27. FOLLENFANT A., MEYER T. Pratiques déclarées, sentiment d'avoir appris et autoefficacité au travail. Résultats de l'enquête quantitative par questionnaires. In: CARRE P., CHARBONNIER O., editor. Les apprentissages professionnels informels. Paris:

L'Harmattan; 2003.

28. PIGUET C. Autonomie dans les pratiques infirmières hospitalières. Contribution à une théorie agentique du développement professionnel. Nanterre, Louvain-la-Neuve: Université Paris-Ouest Nanterre La Défense, Université catholique de Louvain-la-Neuve; 2008.

29. BOURGEOIS É. Motivation et formation des adultes. In: CARRÉ P., FENOUILLET F., editor. Traité de psychologie de la motivation. Paris: Dunod; 2009.

30. ESTRYN-BÉHAR M. Accréditation et qualité des soins hospitaliers. Interface entre les normes et les référentiels et l'activité de travail réelle des soignants Actualité et dossier en santé publique 2001(35).

31. ESTRYN-BÉHAR M., FOUILLOT J.-P. Étude de la charge mentale et approche de la charge physique du personnel soignant. Analyse du travail des infirmières et aides-soignantes dans dix services de soins. Documents pour le médecin du travail. 1990.

32. LEONTIEV A. Le développement du psychisme. Paris: Éditions Sociales; 1976.

33. OMBREDANE A., FAVERGE J.-M. L'analyse du travail. Paris: PUF; 1955.

34. LEPLAT J. L'analyse psychologique de l'activité en ergonomie. Toulouse: Octarès; 2000.

35. PASTRÉ P. Apprendre par la résolution de problèmes : le rôle de la simulation. In: PASTRÉ P., editor. Apprendre par la simulation De l'analyse du travail aux apprentissages professionnels. Toulouse: Octarès; 2005.

36. DE BRITO G., VEYRAC H. Catégorisation des représentations du prescrit et nonrespect des procédures. XXXII Symposium de la Société d'Ergonomie de Langue Française. Lyon; 1997.

37. LEPLAT J. Éléments pour l'étude des documents prescripteurs. @ctivités. 2004;1(2):195-216.

38. MAYEN P., SAVOYANT A. Formation et prescription : une réflexion de didactique professionnelle. In: EVESQUE J-M, GAUTIER A-M., REVEST C., SCHWARTZ Y., VAYSSIERE J-L, editor. Actes du 37ème Congrès - Aix-en-Provence - 2002 "Les évolutions de la prescription": Société d'ergonomie de langue française; 2002.

39. VALOT C. Gestion du temps, gestion du risque (à travers quelques situations aéronautiques). In: CELLIER J-M., De KEYSER V., VALOT C., editor. La gestion du temps dans les environnements dynamiques. Paris: PUF; 1996.

40. MAYEN P. Dix développements sur la didactique professionnelle et le développement. In: LENOIR Y., PASTRÉ P., editor. Didactique professionnelle et didactiques disciplinaires en débat. Toulouse: Octarès; 2008.

41. MAYEN P. Quelques repères pour analyser les situations dans lesquelles le travail consiste à agir pour et avec un autre. Recherches en éducation 2007(4).

42. BAZILLE J, MAYEN P. Le développement des concepts scientifiques à partir des conceptualisations dans l'action. Proposition de didactique professionnelle. ASTER 2002(34). 
43. RABARDEL P., SIX B. Outiller les acteurs de la formation pour le développement des compétences au travail. Éducation permanente. 1995;123 (2).

44. JONNAERT P. Compétences et socioconstructivisme. Bruxelles: De Boeck; 2006. 45. PERRENOUD P. Des savoirs aux compétences. De quoi parle-t-on en parlant de compétences ? Pédagogie collégiale. 1995;9 (1).

46. LEPLAT J. La notion de régulation dans l'analyse de l'activité. Pistes. 2006;8 (1).

47. PASTRÉ P. Apprentissage et activité. In: LENOIR Y., PASTRÉ P., editor. Didactique professionnelle et didactiques disciplinaires en débat. Toulouse: Octarès; 2008.

48. COULET J-C., CHAUVIGNÉ C. Passer d'un référentiel de compétences à une ingénierie de formation. Éducation Permanente. 2005;165 (4).

49. SCHWARZER R., JERUSALEM M. Generalized self-efficacy scale. In: WEINMAN J., WRIGHT S., JOHNSTON M., editor. Measures in health psychology : a user's portfolio Causal and control beliefs. Windsor: NFER - NELSON; 1995. 